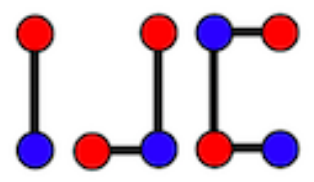

\title{
The forcing monophonic and the forcing geodetic numbers of a graph
}

\author{
J. John \\ Department of Mathematics, Government College of Engineering, \\ Tirunelveli 627 007, India \\ john@gcetly.ac.in
}

\begin{abstract}
For a connected graph $G=(V, E)$, let a set $S$ be a $m$-set of $G$. A subset $T \subseteq S$ is called a forcing subset for $S$ if $S$ is the unique $m$-set containing $T$. A forcing subset for $S$ of minimum cardinality is a minimum forcing subset of $S$. The forcing monophonic number of $S$, denoted by $f_{m}(S)$, is the cardinality of a minimum forcing subset of $S$. The forcing monophonic number of $G$, denoted by $f_{m}(G)$, is $f_{m}(G)=\min \left\{f_{m}(S)\right\}$, where the minimum is taken over all minimum monophonic sets in $G$. We know that $m(G) \leq g(G)$, where $m(G)$ and $g(G)$ are monophonic number and geodetic number of a connected graph $G$ respectively. However there is no relationship between $f_{m}(G)$ and $f_{g}(G)$, where $f_{g}(G)$ is the forcing geodetic number of a connected graph $G$. We give a series of realization results for various possibilities of these four parameters.
\end{abstract}

Keywords: geodetic number, monophonic number, forcing geodetic number, forcing monophonic number Mathematics Subject Classification : 05C12, $05 \mathrm{C} 38$

DOI: $10.19184 /$ ijc.2020.4.2.5

\section{Introduction}

By a graph $G=(V, E)$, we mean a finite undirected connected graph without loops or multiple edges. The order and size of $G$ are denoted by $p$ and $q$ respectively. For basic graph theoretic terminology, we refer to Harary [1]. The distance $d(u, v)$ between two vertices $u$ and $v$ in a connected graph $G$ is the length of shortest $u-v$ path in $G$. An $u-v$ path of length $d(u, v)$ is called an $u-v$

Received: 9 February 2020, Revised: 13 December 2020, Accepted: 26 December 2020. 
geodesic. A vertex $x$ is said to be lie a $u-v$ geodesic $P$ if $x$ is a vertex of $P$ including the vertices $u$ and $v$. A geodetic set of $G$ is a set $S \subseteq V$ such that every vertex of $G$ is contained in geodesic joining some pair of vertices in $S$. The geodetic number $g(G)$ of $G$ is the minimum order of its geodetic sets and any geodetic set of order $g(G)$ is a minimum geodetic set or simply a $g$-set of $G$. The geodetic number of a graph was introduced in [1] and further studied in $[3,4,5,7,8,9,16$, $17,18,20,23,25]$. A subset $T \subseteq S$ is called a forcing subset for $S$ if $S$ is the unique $g$-set of $G$ containing $T$. A forcing subset for $S$ of minimum cardinality is a minimum forcing subset of $S$. The forcing geodetic number of $S$, denoted by $f_{g}(S)$, is the cardinality of a minimum forcing subset of $S$. The forcing geodetic number of $G$, denoted by $f_{g}(G)$, is $f_{g}(G)=\min \left\{f_{g}(S)\right\}$, where the minimum is taken over all minimum $g$-sets of $G$. The forcing geodetic number of a graph was introduced in [3] and furthur studied in [19, 21, 22]. A chord of the path $P$ is an edge joining to non-adjacent vertices of $P$. An $u-v$ path $P$ is called monophonic path if it is a chordless path. A monophonic set of $G$ is a set $S \subseteq V$ such that every vertex of $G$ is contained in a monophonic path joining some pair of vertices in $M$. The monophonic number $m(G)$ of $G$ is the minimum order of its monophonic sets and any monophonic set of order $m(G)$ is a minimum monophonic set or simply a $m$-set of $G$. The monophonic number of a graph was introduced in [6] and further studied in $[2,6,10,11,12,13,14,15,19,24]$. A vertex $v$ is said to be monophonic vertex of $G$ if $v$ belongs to every minimum monophonic set of $G$. A vertex $v$ is an extreme vertex of a graph $G$ if the sub graph induced by its neighbours is complete. A vertex $v$ is said to be geodetic(monophonic) vertex if $v$ belongs to every $g$-set ( $m$-set) of $G$. Every extreme vertices are geodetic(monophonic) vertices of $G$. In fact there are geodetic (monophonic) vertices which are not extreme vertices of $G$. Let $G$ be a connected graph and $S$ a $m$-set of $G$. A subset $T \subseteq S$ is called a forcing subset for $S$ if $S$ is the unique $m$-set of $G$ containing $T$. A forcing subset for $S$ of minimum cardinality is a minimum forcing subset of $S$. The forcing monophonic number of $S$, denoted by $f_{m}(S)$, is the cardinality of a minimum forcing subset of $S$. The forcing monophonic number of $G$, denoted by $f_{m}(G)$ is defined by $f_{m}(G)=\min \left\{f_{m}(S)\right\}$, where the minimum is taken over all $m$-sets $S$ in $G$. The forcing monophonic number of a graph was introduced in [11]. The Throughout the following $G$ denotes a connected graph with at least two vertices. The following theorems are used in the sequel.

Theorem 1.1. $[4,12]$ If $v$ is an extreme vertex of a connected graph $G$, then $v$ belongs to every geodetic (monophonic) set of $G$.

Theorem 1.2. $[1,12]$ For a connected graph $G, g(G)=p(m(G)=p)$ if and only if $G=K_{p}$.

Theorem 1.3. $[3,11]$ Let $G$ be a connected graph, then

a) $f_{g}(G)=0=f_{m}(G)=0$ if and only if $G$ has a unique minimum geodetic (monophonic) set.

b) $f_{g}(G) \leq g(G)-|W|,\left(f_{m}(G) \leq m(G)-|W|\right)$, where $W$ is the set of all geodetic (monophonic) vertices of $G$.

Theorem 1.4. $[3,11]$ For the complete graph $G=K_{p}, f_{g}(G)=f_{m}(G)=0$.

\section{The Forcing Monophonic and the Forcing Geodetic Numbers of a Graph}

We know that $m(G) \leq g(G)$. From the following examples, we observe that there is no relationship between $f_{m}(G)$ and $f_{g}(G)$. 


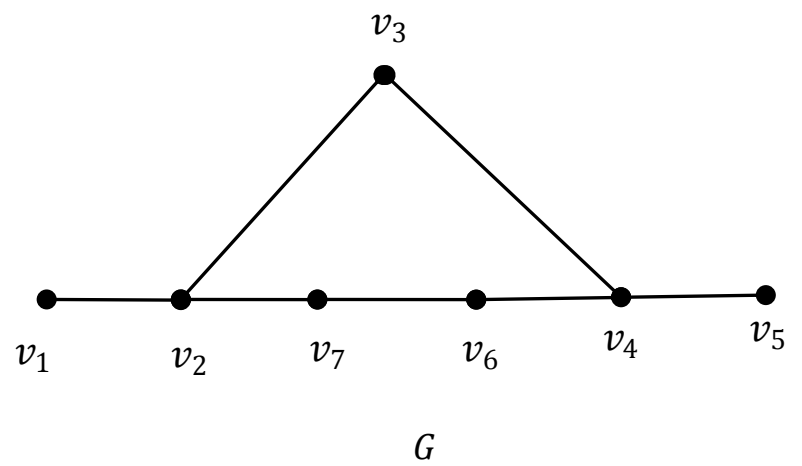

Figure 2.1

Example 2.1. For the graph $G$ given in Figure 2.1, $M=\left\{v_{1}, v_{5}\right\}$ is the unique $m$-set of $G$ so that $f_{m}(G)=0$ and $m(G)=2$. Also $S_{1}=\left\{v_{1}, v_{5}, v_{6}\right\}$ and $S_{2}=\left\{v_{1}, v_{5}, v_{7}\right\}$ are the only two $g$-sets of $G$ such that $f_{g}\left(S_{1}\right)=f_{g}\left(S_{2}\right)=1$ so that $f_{g}(G)=1$ and $g(G)=3$. Thus $f_{m}(G)<f_{g}(G)<m(G)<$ $g(G)$.

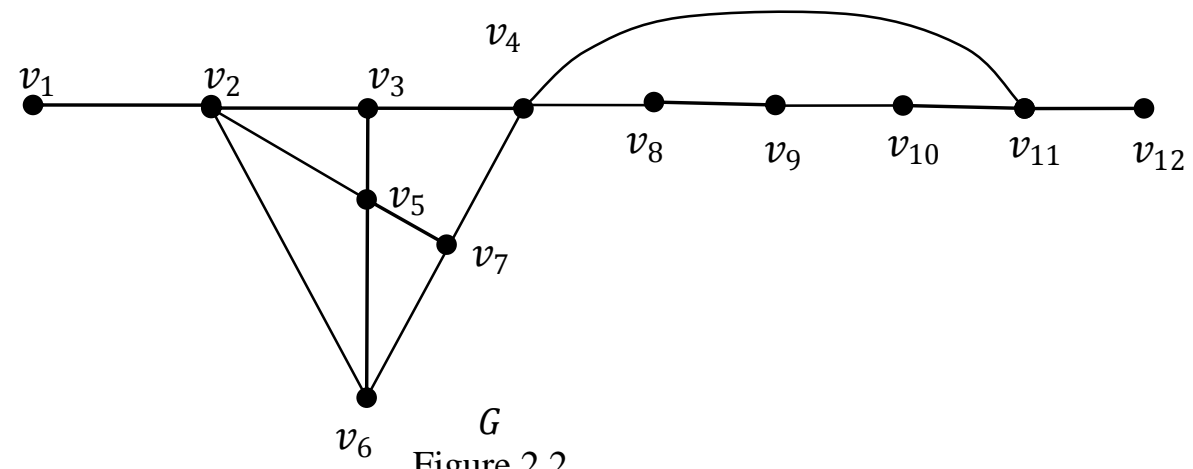

Figure 2.2

Example 2.2. For the graph $G$ given in Figure 2.2, $M_{1}=\left\{v_{1}, v_{8}, v_{12}\right\}, M_{2}=\left\{v_{1}, v_{9}, v_{12}\right\}$ and $M_{3}=\left\{v_{1}, v_{10}, v_{12}\right\}$ are the only three $m$-set of $G$ so that $f_{m}\left(M_{1}\right)=f_{m}\left(M_{2}\right)=f_{m}\left(M_{3}\right)=1$ so that $f_{m}(G)=1$ and $m(G)=3$. Also $S_{1}=\left\{v_{1}, v_{7}, v_{9}, v_{12}\right\}$ is the unique $g$-set of $G$ so that $f_{g}(G)$ $=0$ and $g(G)=4$. Thus $f_{g}(G)<f_{m}(G)<m(G)<g(G)$.

\section{Special graphs}

In this section, we present some graphs from which various graphs arising in theorem are generated using identification.

Let $P_{i}: u_{i}, v_{i}$ be a copy of paths on two vertices. Let $G_{a}$ be the graph given in Figure 3.1 obtained from $P_{i}(\leq i \leq a)$ by introducing new vertices $s, t$ and joining each $u_{i}(1 \leq i \leq a)$ with $s$ and joining each $v_{i}(1 \leq i \leq a)$ with $t$ and join $s$ with $t$. 


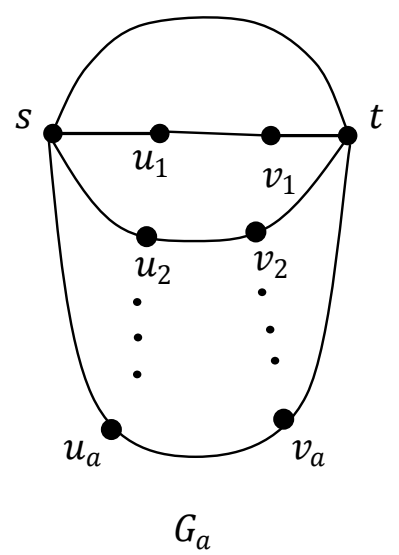

Figure 3.1

Let $P_{i}: n_{i}, p_{i}(1 \leq i \leq b)$ be a copy of path on two vertices and $P: l, m, n$ be a path on three vertices. Let $Z_{b}$ be the graph given in Figure 3.2 obtained from $P_{i}(1 \leq i \leq b)$ and $P$ by joining each $n_{i}(1 \leq i \leq b)$ with $l$, each $p_{i}(1 \leq i \leq b)$ with $q$.

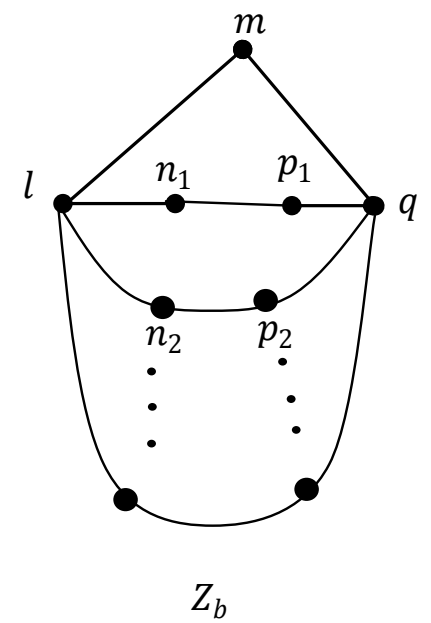

Figure 3.2

Let $P_{i}: r_{i}, h_{i}, k_{i}(1 \leq i \leq c)$ be a copy of path on three vertices and let $P: e, f, g$ be a path on three vertices. Let $H_{c}$ be the graph given in Figure 3.3 obtained from $P_{i}(1 \leq i \leq c)$ and $P$ by joining $e$ and $f$ with each $h_{i}$ and $r_{i}(1 \leq i \leq c)$, joining $g$ with each $k_{i}(1 \leq i \leq c)$, joining $h_{i}$ $(1 \leq i \leq c)$ with $k_{i}(1 \leq i \leq c)$, and joining $r_{i}(1 \leq i \leq c)$ with $k_{i}(1 \leq i \leq c)$. 


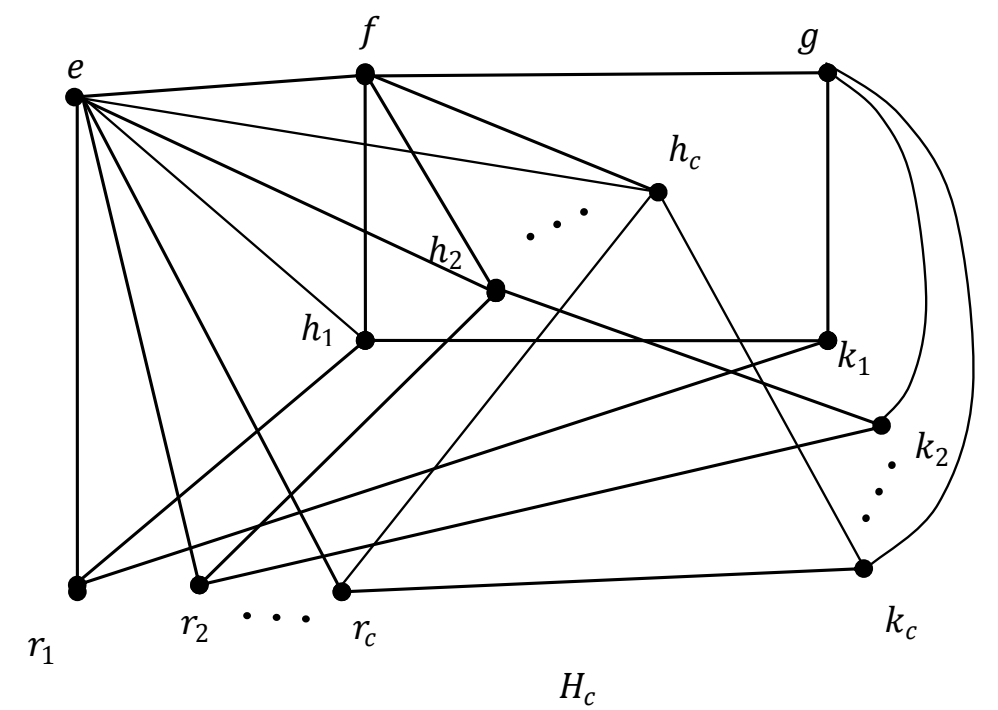

Figure 3.3

Let $U_{i}: x_{i}, y_{i}, w_{i}(1 \leq i \leq d)$ be the path on three vertices. Let $R_{a}$ be the graph given in Figure 3.4 obtained from $U_{i}(1 \leq i \leq d)$ by adding new vertices $u$ and $v$ by joining $u$ with $v$ and joining each $x_{i}(1 \leq i \leq d)$ with $u$ and joining each $w_{i}(1 \leq i \leq d)$ with $v$.

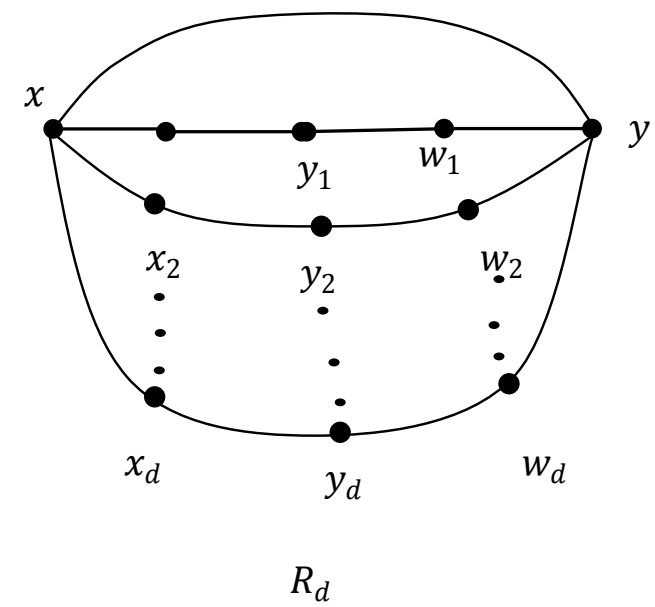

Figure 3.4

\section{Some realization results}

Theorem 4.1. For every pair $a, b$ of integers with $0 \leq a<b$ and $b \geq 2$, there exists a connected graph $G$ such that $f_{m}(G)=f_{g}(G)=0, m(G)=a$ and $g(G)=b$.

Proof. If $a=b$, let $G=K_{a}$. Then by Theorem 1.2, $m(G)=g(G)=a$. Also by Theorem 1.3(a), $f_{m}(G)=f_{g}(G)=0$. For $1 \leq a<b$, let $G$ be the graph obtained from $H_{b-a}$ by adding new 
vertices $x, z_{1}, z_{2}, \ldots, z_{a-1}$ and joining the edges $x e, g z_{1}, g z_{2}, \ldots, g z_{a-1}$. Let $Z=\left\{x, z_{1}, z_{2}, \ldots, z_{a-1}\right\}$ be the set of all end-vertices of $G$. Then it is clear that $Z$ is a monophonic set of $G$ and so by Theorem 1.1, $Z$ is the unique $m$-set of $G$ so that $m(G)=a$ and hence by Theorem 1.3(a), $f_{m}(G)=0$. Since the vertices $h_{i}, k_{i}$ and $r_{i}(1 \leq i \leq b-a)$ does not lie on any geodesic joining a pair of vertices in $Z$, we see that $Z$ is not a geodetic set of $G$. It is easily verified that every $g$-set of $G$ contains each $h_{i}(1 \leq i \leq b-a)$ and so $g(G) \geq b$. Now it is easily seen that $W=Z \cup\left\{h_{1}, h_{2}, \ldots, h_{b-a}\right\}$ is the unique $g$-set of $G$ and hence by Theorem 1.1 and Theorem 1.3(a) $g(G)=b$ and $f_{g}(G)=0$.

Theorem 4.2. For every integers $a, b$ and $c$ with $0 \leq a<b<c$ and $c>a+b$, there exists a connected graph $G$ such that $f_{m}(G)=0, f_{g}(G)=a, m(G)=b$ and $g(G)=c$.

Proof. Case 1. $a=0$. Then the graph $G$ constructed in Theorem 4.1 satisfies the requirements of this theorem.

Case 2. $a \geq 1$. Let $G$ be the graph obtained from $Z_{a}$ and $H_{c-(a+b)}$ by identifying the vertex $q$ of $Z_{a}$ and $e$ of $H_{c-(a+b)}$ and then adding new vertices $x, z_{1}, z_{2}, \ldots, z_{b-1}$ and joining the edges $x l, g z_{1}, g z_{2}, \ldots, q z_{b-1}$. It is clear that $Z$ is a monophonic set of $G$ and by Theorem $1.1, Z$ is the unique $m$-set of $G$ so that $m(G)=b$ and hence by Theorem 1.3(a), $f_{m}(G)=0$. Next we show that $g(G)=c$. Let $S$ be any geodetic set of $G$. Then by Theorem $1.1, Z \subseteq S$. It is clear that $Z$ is not a geodetic set of $G$. For $1 \leq i \leq a$, let $Q_{i}=\left\{n_{i}, p_{i}\right\}$. We observed that every $g$-set of $G$ must contain at least one vertex from each $Q_{i}(1 \leq i \leq a)$ and each $h_{i}(1 \leq i \leq c-b-a)$ so that $g(G) \geq b+a+c-a-b=c$. Now $W=Z \cup\left\{h_{1}, h_{2}, \ldots, h_{c-a-b}\right\} \cup\left\{n_{1}, n_{2}, \ldots, n_{a}\right\}$ is a geodetic set of $G$ so that $g(G) \geq b+a+c-a-b=c$. Thus $g(G)=c$. Since every $g$ - set contains $W_{1}=Z \cup\left\{h_{1}, h_{2}, \ldots, h_{c-a-b}\right\}$ it follows from that from Theorem 1.3 (b) that $f_{g}(G) \leq g(G)-\left|W_{1}\right|=c-(c-a)=a$. Now, since $g(G)=c$ and every $g$-set of $G$ contains $W_{1}$, it is easily seen that every $g$-set $S$ is of the form $W_{1} \cup\left\{d_{1}, d_{2}, \ldots, d_{a}\right\}$ where $d_{i} \in Q_{i}(1 \leq i \leq a)$. Let $T$ be any proper subset of $S$ with $|T|<a$. Then it is clear that there exists some $j$ such that $T \cap Q_{j}=\Phi$, which shows that $f_{g}(G)=a$.

Theorem 4.3. For every integers $a, b$ and $c$ with $0 \leq a<b \leq c$ and $b>a+1$ there exists a connected graph $G$ such that $f_{g}(G)=0, f_{m}(G)=a, m(G)=b$ and $g(G)=c$.

Proof. Case 1. $a=0$. Then the graph $G$ constructed in Theorem 4.1 satisfies the requirements of this theorem.

Case 2. $a \geq 1$.

Subcase 2a. $b=c$. Let $G$ be the graph obtained from $R_{a}$ by adding new vertices $x, z_{1}, z_{2}, \ldots$, $z_{b-a-1}$ and joining the edges $x u, v z_{1}, v z_{2}, \ldots, v z_{b-a-1}$. Let $Z=\left\{x, z_{1}, z_{2}, \ldots, z_{b-a-1}\right\}$ be the set of all end-vertices of $G$. Let $S$ be any geodetic set of $G$. Then by Theorem 1.1, $Z \subseteq S$. It is clear that $Z$ is not a geodetic set of $G$. For $1 \leq i \leq a$, let $H_{i}=\left\{x_{i}, y_{i}, w_{i}\right\}$. We observe that every $g$-set of $G$ must contain only the vertex $y_{i}$ from each $H_{i}(1 \leq i \leq a)$ and so $g(G) \geq b-a+a=b$. Now $S=Z \cup\left\{y_{1}, y_{2}, y_{3}, \ldots, y_{a}\right\}$ is a geodetic set of $G$ so that $g(G) \leq b-a+a=b$. Thus $g(G)=b$. Also it is easily seen that $W$ is the unique $g$-set of $G$ and so $f_{g}(G)=0$. Now it is clear that $Z$ is not a monophonic set of $G$. We observe that every $m$-set of $G$ must contain at least one vertex from each $H_{i}(1 \leq i \leq a)$. Hence by Theorem 1.1, $m(G) \geq b-a+a=b$. Now 
$W_{1}=Z \cup\left\{y_{1}, y_{2}, y_{3}, \ldots, y_{a}\right\}$ is a monophonic set of $G$ so that $m(G) \leq b-a+a=b$. Thus $m(G)=b$. Next we show that $f_{m}(G)=a$. Since every $m$-set contains $Z$, it follows from Theorem 1.3 (b) that $f_{m}(G) \leq m(G)-|Z|=b-(b-a)=a$. Now, since $m(G)=b$ and every $m$-set of $G$ contains $Z$, it is easily seen that every $m$-set $S$ is of the form $Z \cup\left\{d_{1}, d_{2}, d_{3}, \ldots, d_{a}\right\}$, where $d_{i} \in H_{i}(1 \leq i \leq a)$. Let $T$ be any proper subset of $S$ with $|T|<a$. Then it is clear that there exists some $j$ such that $T \cap H_{j}=\Phi$, which shows that $f_{m}(G)=a$.

Subcase 2b. $b<c$. Let $G$ be the graph obtained from $R_{a}$ and $H_{c-b}$ by identifying the vertex $v$ of $R_{a}$ and $g$ of $H_{c-b}$ and then adding the new vertices $x, z_{1}, z_{2}, \ldots, z_{b-a-1}$ and joining the edges $x u, g z_{1}, g z_{2}, \ldots, g z_{b-a-1}$. Let $Z=\left\{x, z_{1}, z_{2}, \ldots, z_{b-a-1}\right\}$ be the set of end vertices of $G$. Let $S$ be any geodetic set of $G$. Then by Theorem $1.1 Z \in S$. It is clear that $Z$ is not a geodetic set of $G$. For $1 \leq i \leq a$, let $H_{i}=\left\{x_{i}, y_{i}, w_{i}\right\}$. We observe that every $g$-set of $G$ must contain only the vertex $y_{i}(1 \leq i \leq a)$ from each $H_{i}(1 \leq i \leq a)$ and each $h_{i}(1 \leq i \leq c-b)$ and so $g(G) \geq b-a+a+c-b=c$ Now $W=Z \cup\left\{y_{1}, y_{2}, y_{3}, \ldots, y_{a}\right\} \cup\left\{h_{1}, h_{2}, h_{3}, \ldots, h_{c-b}\right\}$ is a geodetic set of $G$ so that $g(G) \leq b-a+a+c-b=c$. Thus $g(G)=c$. Also it is easily seen that $W$ is the unique $g$-set of $G$ and so $f_{g}(G)=0$. It is clear that $Z$ is not a monophonic set of $G$. We observe that every $m$-set of $G$ must contain at least one vertex from each $H_{i}(1 \leq i \leq a)$ and so $m(G) \geq b-a+a=b$. Now, $S_{1}=Z \cup\left\{y_{1}, y_{2}, y_{3}, \ldots, y_{a}\right\}$ is a monophonic set of $G$ so that $m(G) \leq b-a+a=b$. Thus $m(G)=b$. Next we show that $f_{m}(G)=a$. Since every $m$-set contains $Z$, it follows from Theorem 1.3 (b) that $f_{m}(G) \leq m(G)-|Z|=b-(b-a)=a$. Now, since $m(G)=b$ and every $m$-set of $G$ contains $Z$, it is easily seen that every $m$-set $S$ is of the form $Z \cup\left\{d_{1}, d_{2}, d_{3}, \ldots, d_{a}\right\}$, where $d_{i} \in H_{i}(1 \leq i \leq a)$. Let $T$ be any proper subset of $S$ with $|T|<a$. Then it is clear that there exists some $j$ such that $T \cap H_{j}=\Phi$, which shows that $f_{m}(G)=a$.

Theorem 4.4. For every pair $a, b$ and $c$ of integers with $0 \leq a \leq b \leq c, b>a+1$ there exists a connected graph $G$ such that $f_{g}(G)=f_{m}(G)=a, m(G)=b$ and $g(G)=c$.

Proof. Case 1. $a=0$, then the graph $G$ constructed in Theorem 4.1 satisfies the requirements of this theorem.

Case 2. $a \geq 1$,

Subcase 2a. $b=c$. Let $G$ be the graph obtained from $G_{a}$ by adding new vertices $x, z_{1}, z_{2}, \ldots$, $z_{b-a-1}$ and joining the edges $x s, t z_{1}, t z_{2}, \ldots, t z_{b-a-1}$. Let $Z=\left\{x, z_{1}, z_{2}, \ldots, z_{a-b-1}\right\}$ be the set of end-vertices of $G$. First we show that $m(G)=b$. Let $M$ be any monophonic set of $G$. Then by Theorem 1.1, $Z \subseteq M$. It is clear that $Z$ is not a monophonic set of $G$. Let $F_{i}=\left\{u_{i}, v_{i}\right\}$ $(1 \leq i \leq a)$. We observe that every $m$-set of $G$ must contain at least one vertex from each $F_{i}$ $(1 \leq i \leq a)$. Thus $m(G) \geq b-a+a=b$. On the other hand since the set $W=Z \cup\left\{v_{1}, v_{2}, \ldots, v_{a}\right\}$ is a monophonic set of $G$, it follows that $m(G) \leq|W|=b$. Hence $m(G)=b$. Next we show that $f_{m}(G)=a$. By Theorem 1.1, every monophonic set of $G$ contains $Z$ and so it follows from Theorem 1.3(b) that $f_{m}(G) \leq m(G)-|Z|=a$. Now, since $m(G)=b$ and every $m$-set of $G$ contains $Z$, it is easily seen that every $m$-set $S$ is of the form $Z \cup\left\{c_{1}, c_{2}, \ldots, c_{a}\right\}$, where $c_{i} \in F_{i}$ $(1 \leq i \leq a)$. Let $T$ be any proper subset of $S$ with $|T|<a$. Then it is clear that there exists some $j$ such that $T \cap H_{j}=\Phi$, which shows that $f_{m}(G)=a$. By similar way we can prove $g(G)=b$ and $f_{g}(G)=a$.

Subcase 2b. $b<c$. Let $G$ be the graph obtained from $G_{a}$ and $H_{c-b}$ by identifying the vertex $t$ of 
$G_{a}$ and the vertex $e$ of $H_{c-b}$ and then adding the new vertices $x, z_{1}, z_{2}, \ldots, z_{b-a-1}$ and joining the edges $x s, g z_{1}, g z_{2}, \ldots, g z_{b-a-1}$.

First we show that $m(G)=b$. Let $Z=\left\{z_{1}, z_{2}, \ldots, z_{b-a-1}\right\}$ be the set of all end-vertices of $G$. Since the vertices $u_{i}, v_{i}$ do not lie on any monophonic path joining a pair of vertices of $Z$, it is clear that $Z$ is not a monophonic set of $G$. Let $F_{i}=\left\{u_{i}, v_{i}\right\}(1 \leq i \leq a)$. We observe that every $m$-set of $G$ must contain at least one vertex from each $F_{i}(1 \leq i \leq a)$. Thus $m(G) \geq$ $b-a+a=b$. On the other hand since the set $W=Z \cup\left\{v_{1}, v_{2}, v_{3}, \ldots, v_{a}\right\}$ is a monophonic set of $G$, it follows that $m(G) \leq|W|=b$. Hence $m(G)=b$. Next, we show that $f_{m}(G)=a$. By Theorem 1.1, every monophonic set of $G$ contains $Z$ and so it follows from Theorem 1.3 (b) that $f_{m}(G) \leq m(G)-|Z|=a$. Now, since $m(G)=b$ and every $m$-set of $G$ contains $Z$, it is easily seen that every $m$-set $S$ is of the form $Z \cup\left\{c_{1}, c_{2}, c_{3}, \ldots, c_{a}\right\}$, where $c_{i} \in F_{i}(1 \leq i \leq a)$. Let $T$ be any proper subset of $S$ with $|T|<a$. Then it is clear that there exists some $j$ such that $T \cap H_{j}=\Phi$, which shows that $f_{m}(G)=a$. Next we show that $g(G)=c$. Since the vertices $u_{i}, v_{i}, h_{i}(1 \leq i \leq a)$ do not lie on any geodesic joining a pair of vertices of $Z$, it is clear that $Z$ is not a geodetic set of $G$. We observe that every $g$-set of $G$ must contain each $H_{i}(1 \leq i \leq a)$ and each $h_{i}(1 \leq i \leq c-b)$ so that $g(G) \geq b-a+a+c-b=c$. On the other hand, since the set $S_{1}=Z \cup\left\{h_{1}, h_{2}, h_{3}, \ldots, h_{c-b}\right\} \cup\left\{u_{1}, u_{2}, \ldots, u_{a}\right\}$ is a geodetic set of $G$, so that $g(G) \leq\left|S_{1}\right|=c$. Hence $g(G)=c$. Next we show that $f_{g}(G)=a$. By Theorem 1.1, every geodetic set of $G$ contains $W_{1}=Z \cup\left\{h_{1}, h_{2}, h_{3}, \ldots, h_{c-b}\right\}$ and so it follows from Theorem 1.3(b) that $f_{g}(G) \leq g(G)-\left|W_{1}\right|=a$. Now, since $g(G)=c$ and every $g$-set of $G$ contains $Z$, it is easily seen that every $g$-set $S$ is of the form $W_{1} \cup\left\{c_{1}, c_{2}, c_{3}, \ldots, c_{a}\right\}$, where $c_{i} \in F_{i}(1 \leq i \leq a)$. Let $T$ be any proper subset of $S$ with $|T|<a$. Then it is clear that there exists some $j$ such that $T \cap H_{j}=\Phi$, which shows that $f_{g}(G)=a$. This is true for all $g$-sets of $G$ so that $f_{g}(G)=a$.

Theorem 4.5. For every integers $a, b, c$ and $d$ with $2 \leq c<d, 0 \leq a \leq b \leq d$ and $d>c-a+b$, there exists a connected graph $G$ such that $f_{m}(G)=a, f_{g}(G)=b, m(G)=c$ and $g(G)=d$.

Proof. Case 1. $a=b=0$. Then the graph $G$ constructed in Theorem 4.1 satisfies the requirements of this theorem.

Case 2. $a=0, b>1$. Then the graph $G$ constructed in Theorem 4.2 satisfies the requirements of this theorem.

Case 3. $1 \leq a=b$. Then the graph $G$ constructed in Theorem 4.4 satisfies the requirements of this theorem.

Case 4. $1 \leq a<b$. Let $G_{1}$ be the graph obtained from $G_{a}$ and $Z_{b-a}$ by identifying the vertex $t$ of $G_{a}$ and the vertex $l$ of $Z_{b-a}$. Now let $G$ be the graph obtained from $G_{1}$ and $H_{d-(c-a+b)}$ by identifying the vertex $q$ of $G_{1}$ and the vertex $e$ of $H_{d-(c-a+b)}$ and adding new vertices $x, z_{1}, z_{2}, \ldots, z_{c-a-1}$ and joining the edges $x s, g z_{1}, g z_{2}, \ldots, g z_{c-a-1}$. Let $Z=\left\{x, z_{1}, z_{2}, \ldots, z_{c-a-1}\right\}$ be the set of end vertices of $G$. For $1 \leq i \leq a$ let $F_{i}=\left\{u_{i}, v_{i}\right\}$. It is clear that any $m$-set is of the form $S=Z \cup\left\{c_{1}, c_{2}, c_{3}, \ldots, c_{a}\right\}$ where $c_{i} \in F_{i}(1 \leq i \leq a)$. Then as in earlier theorems it can be seen that $f_{m}(G)=a$ and $m(G)=c$. For $1 \leq i \leq a$ let $Q_{i}=\left\{n_{i}, p_{i}\right\}$. It is clear that any $g$-set is of the form $W=Z \cup\left\{h_{1}, h_{2}, h_{3}, \ldots, h_{d-(c-a+b)}\right\} \cup\left\{c_{1}, c_{2}, c_{3}, \ldots, c_{a}\right\} \cup\left\{d_{1}, d_{2}, d_{3}, \ldots, d_{b-a}\right\}$, where $c_{i} \in F_{i}(1 \leq i \leq a)$ and $d_{j} \in Q_{j}(1 \leq j \leq b-a)$. Then as in earlier theorems it can be seen that $f_{g}(G)=b$ and $g(G)=d$. 
Theorem 4.6. For every integers $a, b, c$ and $d$ with $0 \leq a \leq b<c \leq d$ and $c \geq b+1$ and $c, d \geq 2$ there exists a connected graph $G$ such that $f_{g}(G)=a, f_{m}(G)=b, m(G)=c$ and $g(G)=d$.

Proof. Case 1. $a=b=0$. Then the graph $G$ constructed in Theorem 4.1 satisfies the requirements of this theorem.

Case 2. $a=0, b \geq 1$. Then the graph $G$ constructed in Theorem 4.2 satisfies the requirements of this theorem.

Case 3. $1 \leq a=b$. Then the graph $\mathrm{G}$ constructed in Theorem 4.4 satisfies the requirements of this theorem.

Case 4. $1 \leq a<b$.

Subcase 4a. $c=d$. Let $G$ be the graph obtained from $G_{a}$ and $R_{b-a}$ by identifying the vertex $t$ of $G_{a}$ and the vertex $q$ of $R_{b-a}$ and then adding the new vertices $x, z_{1}, z_{2}, \ldots, z_{c-b-1}$ and joining the edges $x s, q z_{1}, q z_{2}, \ldots, q z_{c-b-1}$. First we show that $m(G)=c$. Let $Z=\left\{x, z_{1}, z_{2}, \ldots, z_{c-b-1}\right\}$ be the set of end vertices of $G$. Let $F_{i}=\left\{u_{i}, v_{i}\right\}(1 \leq i \leq a)$ and $H_{i}=\left\{x_{i}, y_{i}, w_{i}\right\}(1 \leq i \leq b-a)$. It is clear that any $m$-set of $G$ is of the form $S=Z \cup\left\{c_{1}, c_{2}, c_{3}, \ldots, c_{a}\right\} \cup\left\{d_{1}, d_{2}, d_{3}, \ldots, d_{b-a}\right\}$ where $c_{i} \in F_{i}(1 \leq i \leq a)$ and $d_{j} \in H_{j}(1 \leq j \leq b-a)$. Then as in earlier theorems it can be seen that $f_{m}(G)=b$ and $m(G)=c$. It is clear that any $g$-set is of the form $W=$ $Z \cup\left\{y_{1}, y_{2}, y_{3}, \ldots, y_{b-a}\right\} \cup\left\{c_{1}, c_{2}, c_{3}, \ldots, c_{a}\right\}$, where $c_{i} \in F_{i}(1 \leq i \leq a)$. Then as in earlier theorems it can be seen that $f_{g}(G)=a$ and $m(G)=c$.

Subcase 4b. $c<d$. Let $G_{1}$ be the graph obtained from $G_{a}$ and $R_{b-a}$ by identifying the vertex $t$ of $G_{a}$ and the vertex $v$ of $R_{b-a}$. Now let $G$ be the graph obtained from $G_{1}$ and $Z_{d-c}$ by identifying the vertex $q$ of $G_{1}$ and the vertex $l$ of $Z_{d-c}$ and then adding new vertices $x, z_{1}, z_{2}, \ldots, z_{c-b-1}$ and joining the edges $x s, q z_{1}, q z_{2}, \ldots, q z_{c-b-1}$. Let $Z=\left\{x, z_{1}, z_{2}, \ldots, z_{c-b-1}\right\}$ be the set of end vertices of $G$. Let $F_{i}=\left\{u_{i}, v_{i}\right\}(1 \leq i \leq a)$ and $H_{i}=\left\{x_{i}, y_{i}, w_{i}\right\}(1 \leq i \leq b-a)$. It is clear that any $m$-set of $G$ is of the form $S=Z \cup\left\{c_{1}, c_{2}, c_{3}, \ldots, c_{a}\right\} \cup\left\{d_{1}, d_{2}, d_{3}, \ldots, d_{b-a}\right\}$ where $c_{i} \in F_{i}(1 \leq i \leq a)$ and $d_{j} \in H_{j}(1 \leq j \leq b-a)$. Then as in earlier theorems it can be seen that $f_{m}(G)=b$ and $m(G)=c$. It is clear that any $g$-set is of the form $W=$ $Z \cup\left\{y_{1}, y_{2}, y_{3}, \ldots, y_{b-a}\right\} \cup\left\{h_{1}, h_{2}, h_{3}, \ldots, h_{d-c}\right\} \cup\left\{c_{1}, c_{2}, c_{3}, \ldots, c_{a}\right\}$ where $c_{i} \in F_{i}(1 \leq i \leq a)$. Then as in earlier theorems it can be seen that $f_{g}(G)=a$ and $g(G)=d$.

In the realiztion results we have given some restrictions on the parameters. So we leave the following as open question.

Problem 1. For any four positive integers $a, b, c$ and $d$ with $a \geq 0, b \geq 0$ and $2 \leq c \leq d$, does there exists a connected graph $G$ with $f_{m}(G)=a, f_{g}(G)=b, m(G)=c$ and $g(G)=d$.

\section{The Upper Forcing Monophonic number of a graph}

In [25], P. Zhang introduced the concept of the upper geodetic number of a graph. In the similar manner we define the upper forcing monophonic number of a graph as follows.

Definition 5.1. Let $G$ be a connected graph and $S$ a $m$-set of $G$. A subset $T \subseteq S$ is called a forcing subset for $S$ if $S$ is the unique $m$-set containing $T$. A forcing subset for $S$ of minimum cardinality is a minimum forcing subset of $S$. The forcing monophonic number of $S$, denoted by $f_{m}(S)$, is 
the cardinality of a minimum forcing subset of $S$. The forcing monophonic number of $G$, denoted by $f_{m}(G)$ is defined by $f_{m}(G)=\min \left\{f_{m}(S)\right\}$, where the minimum is taken over all $m$-set $S$ in $G$ and the upper forcing monophonic number of $G$, denoted by $f_{m}^{+}(G)=\max \left\{f_{m}(S)\right\}$, where the maximum is taken over all $m$-sets $S$ in $G$.

Theorem 5.2. For every connected graph $G, 0 \leq f_{m}(G) \leq f_{m}^{+}(G) \leq m(G)$.

Example 5.3. The bounds in Theorem 5.2 is sharp. For $G=K_{1, p-1}, f_{m}(G)=0$. For $G=C_{5}$, $f_{m}(G)=f_{m}^{+}(G)=2$. Also the inequalities in Theorem 5.2 can be strict. For the graph $G$ given in Figure 5.1, $M_{1}=\left\{v_{1}, v_{4}, v_{5}\right\}, M_{2}=\left\{v_{1}, v_{4}, v_{6}\right\}$ and $M_{1}=\left\{v_{1}, v_{3}, v_{5}\right\}$ are only three $m$-sets of $G$ so that $f_{m}\left(M_{1}\right)=2, f_{m}\left(M_{2}\right)=1$ and $f_{m}\left(M_{3}\right)=2$ so that $f_{m}(G)=2, f_{m}^{+}(G)=2$ and $m(G)=3$. Thus $0<f_{m}(G)<f_{m}^{+}(G)<m(G)$.

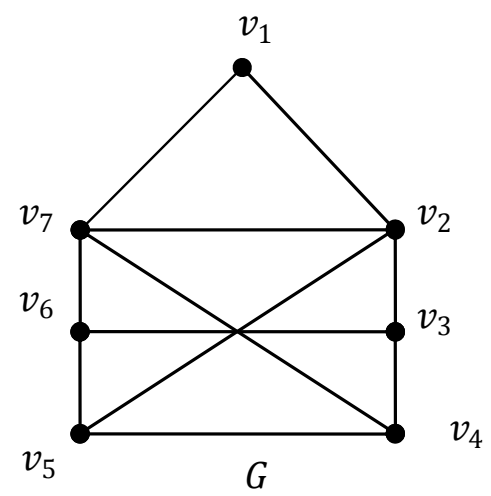

Figure 5.1

So we leave the following as a open question.

Problem 2. For any three positive integers $a, b$ and $c$ with $0 \leq a \leq b \leq c$, does there exists $a$ connected graph $G$ with $f_{m}(G)=a, f_{m}^{+}(G)=b$ and $m(G)=c$.

\section{References}

[1] H.A. Ahangara, S. Kosarib, S.M. Sheikholeslamib, and L. Volkmannc, Graphs with large geodetic number, Filomat. 29:6 (2015), 1361 - 1368.

[2] F. Buckley and F. Harary, Distance in Graphs, Addison-Wesley, Redwood City, CA, 1990.

[3] J. Caceres, O. Oellermann, and M. Puertasa, Minimal trees and monophonic convexity, Discuss. Math. Graph Theory, 32, (2012), 685 - 704.

[4] G. Chartrand and P. Zhang, The forcing geodetic number of a graph, Discuss. Math. Graph Theory, 19, (1999), $45-58$. 
[5] G. Chartrand, F. Harary, and P. Zhang, On the geodetic number of a graph, Networks, (2002), $1-6$.

[6] M.C. Dourado, F. Protti, D. Rautenbach, and J.L. Szwarcfiter, Some remarks on the geodetic number of a graph, Discrete Math. 310, (2010), 832 - 837.

[7] M.C. Dourado, F. Protti, and J.L. Szwarcfiter, Algorithmic aspects of monophonic convexity, Electron. Notes Discrete Math. 30, (2008), 177 - 182.

[8] F. Harary, E. Loukakis, and C. Tsouros, The geodetic number of a graph, Math. Comput. Modeling, 17(11), (1993), 89 - 95.

[9] C. Hernando, T. Jiang, M. Mora, I.M. Pelayo, and C. Seara, On the Steiner, geodetic and hull number of graphs, Discrete Math. 293, (2005), 139 - 154.

[10] J. John and P.A.P. Sudhahar, The forcing edge monophonic number of a graph , SCIENTIA Series A: Mathematical Sciences, 23, (2012), 87-98

[11] J. John and S. Panchali, The forcing monophonic number of a graph, IJMA-3 (3), (2012), 935 $-938$.

[12] J. John and S. Panchali, The upper monophonic number of a graph, Int. J. Math. Combin. 4, (2010), $46-52$

[13] J. John and K.U. Samundeswari, The forcing edge fixing edge-to-vertex monophonic number of a graph, Discrete Math. Algorithms Appl. 5(4), (2013), 1 - 10.

[14] J. John and K.U. Samundeswari, The edge fixing edge-to-vertex monophonic number of a graph, Appl. Math. E-Notes, 15, (2015), $261-275$.

[15] J. John and K.U. Samundeswari, Total and forcing total edge-to-vertex monophonic numbers of graph, J. Comb. Optim. 34, (2017), 1 - 14.

[16] J. John and D. Stalin, Edge geodetic self decomposition in graphs, Discrete Math. Algorithms Appl. 12(5), (2020), 2050064, 7 pages.

[17] J. John and D. Stalin, The edge geodetic self decomposition number of a graph, RAIRO Oper. Res. , DOI:10.1051/ro/2020073.

[18] J. John and D. Stalin, Distinct edge geodetic decomposition in graphs, Commun. Comb. Optim., DOI: 10.22049/CCO.2020.26638.1126

[19] E.M. Paluga and S.R. Canoy, Jr, Monophonic numbers of the join and composition of connected graphs, Discrete Math. 307, (2007), 1146 - 1154.

[20] I.M. Pelayo, Geodesic Convexity in Graphs, Springer Briefs in Mathematics, 2013. 
[21] A.P. Santhakumaran and J. John, On the forcing geodetic and forcing Steiner numbers of a graph, Discuss. Math. Graph Theory, 31, (2011), 611 - 624.

[22] Li-Da Tong, Geodetic sets and Steiner sets in graphs, Discrete Math. 309(12), (2009), 3733 $-4214$.

[23] Li-Da Tong, The forcing hull and forcing geodetic numbers of graphs, Discrete Appl. Math. 157(5), (2009), 875 - 1164.

[24] Li-Da Tong, The (a,b)-forcing geodetic graphs, Discrete Math. 309(6), (2009), 1199 - 1792.

[25] P. Zhang, The upper forcing geodetic number of a graph, Ars Combin., DOI: 10.7151/dmgt.1084. 